\title{
Adenoma pleomórfico em mucosa jugal: relato de caso
}

\author{
Pleomorphic adenoma in jugal mucosa: case report \\ Adenoma pleomórfico en mucosa yugal: reporte de un caso
}

Flávia de Paiva Teixeira Barros

ORCID: https://orcid.org/0000-0001-6545-9021

Centro Universitário Cesmac, Brasil

E-mail: flaviapaivatbarros@gmail.com

Gabrielly Maria Argolo Acioly

ORCID: https://orcid.org/0000-0003-4248-7892

Centro Universitário Cesmac, Brasil

E-mail: Gaabiargolo@hotmail.com

Camila Holanda Cavalcante Matos

ORCID: https://orcid.org/0000-0001-8283-0590

Centro Universitário Cesmac, Brasil

E-mail: camilaacavalcantem@outlook.com

Carla Beatriz dos Anjos Mendes

ORCID: https://orcid.org/0000-0003-4090-7451

Centro Universitário Cesmac, Brasil

E-mail: carlabmendes@hotmail.com

Renata Cordeiro de Godoy Miranda

ORCID: https://orcid.org/0000-0002-7224-4173

Centro Universitário Cesmac, Brasil

E-mail: renatagodoymiranda@hotmail.com

Beatriz Nunes Lins

ORCID: https://orcid.org/0000-0003-0244-7140 Centro Universitário Cesmac, Brasil E-mail: bianuneslins@gmail.com

Gabriel Alencar Lemos de Alcântara

ORCID: https://orcid.org/0000-0002-9932-9687 Centro Universitário Cesmac, Brasil

E-mail: gabriel-morais12@hotmail.com

Taj Mahal Ferreira Wanderley Santos ORCID: https://orcid.org/0000-0003-1993-2900 Centro Universitário Cesmac, Brasil E-mail: tajmahalw@hotmail.com

Áurea Valéria de Melo Franco

ORCID: https://orcid.org/0000-0002-5966-8617 Centro Universitário Cesmac, Brasil E-mail: aurea.franco@cesmac.edu.br

Camila Maria Beder Ribeiro Girish Panjwani ORCID: https://orcid.org/0000-0003-0203-3079 Centro Universitário Cesmac, Brasil

E-mail: camila.panjwani@cesmac.edu.br

Vanessa de Carla Batista dos Santos

ORCID: https://orcid.org/0000-0002-3267-3969 Centro Universitário Cesmac, Brasil

E-mail: vanessadecarlla@yahoo.com.br

Fernanda Braga Peixoto

ORCID: https://orcid.org/0000-0003-4549-4959 Centro Universitário Cesmac, Brasil

E-mail: fernanda.peixoto@cesmac.edu.br

\section{Resumo}

Objetivo: Destacar a relevância diagnóstica na prática odontológica clínica, buscando a correta conduta no tratamento do adenoma pleomórfico em região de mucosa jugal, bem como discutir a patogênese da lesão. Detalhamento do caso: Paciente do sexo masculino, 64 anos, compareceu a Clínica Escola de Odontologia queixando-se de lesão assintomática que surgiu de forma lenta há aproximadamente 8 meses. Ao exame intraoral foi observada uma lesão nodular e única em mucosa jugal, de coloração branca, avermelhada e amarelada, superfície lisa, bem delimitada, de formato oval e consistência firme. A hipótese de diagnóstico foi de lipoma. Optou-se pela biópsia excisional para confirmação da lesão através do estudo anatomopatológico. Considerações finais: A realização da biópsia é imprescindível, a fim descartar 
outras hipóteses diagnósticas. É importante que o profissional cirurgião-dentista tenha capacitação técnica para propor o tratamento mais adequado, devolvendo bem-estar, autoestima e saúde do paciente, enquanto mantém um prognóstico satisfatório.

Palavras-chave: Adenoma pleomórfico; Mucosa jugal; Patologia oral.

\begin{abstract}
Objective: Highlight the diagnostic relevance in clinical dental practice, seeking the correct approach in the treatment of pleomorphic adenoma in the region of the buccal mucosa, as well as discussing the pathogenesis of the lesion. Case details: Male patient, 64 years old, attended the Dental School Clinic complaining of an asymptomatic lesion that appeared slowly approximately 8 months ago. The intraoral examination revealed a single nodular lesion in the buccal mucosa, white, reddish and yellowish in color, smooth, well-delimited, oval-shaped and firm consistency. The diagnosis hypothesis was lipoma. We opted for excisional biopsy to confirm the lesion through anatomopathological study. Final considerations: The performance of biopsy is essential in order to rule out other diagnostic hypotheses. It is important that the dental surgeon has technical training to propose the most appropriate treatment, restoring the patient's wellbeing, self-esteem and health, while maintaining a satisfactory prognosis.
\end{abstract}

Keywords: Pleomorphic adenoma; Buccal mucosa; Oral pathology.

\title{
Resumen
}

Objetivo: Destacar la relevancia diagnóstica en la práctica clínica odontológica, buscando el abordaje correcto en el tratamiento del adenoma pleomórfico en la región de la mucosa bucal, así como discutir la patogenia de la lesión. Detalles del caso: Paciente masculino de 64 años que acudió a la Clínica Facultad de Odontología por lesión asintomática de aparición lenta hace aproximadamente 8 meses. El examen intraoral reveló una única lesión nodular en la mucosa bucal, de color blanco, rojizo y amarillento, lisa, bien delimitada, ovalada y de consistencia firme. La hipótesis diagnóstica fue lipoma. Se optó por una biopsia excisional para confirmar la lesión mediante estudio anatomopatológico. Consideraciones finales: La realización de biopsia es fundamental para descartar otras hipótesis diagnósticas. Es importante que el cirujano dental cuente con la formación técnica para proponer el tratamiento más adecuado, devolviendo el bienestar, la autoestima y la salud del paciente, manteniendo un pronóstico satisfactorio.

Palabras clave: Adenoma pleomórfico; Mucosa bucal; Patología bucal.

\section{Introdução}

O complexo buco-maxilo-facial pode apresentar uma série de lesões císticas e tumorais, dentre elas, existem os tumores de origem das glândulas salivares. O Adenoma pleomórfico (AP) é o tumor benigno mais frequente das glândulas salivares, cerca de 70 a $90 \%$ dos casos (Silva et al., 2007).

O adenoma pleomórfico apresenta predileção pelo sexo feminino, com prepoderância dos 30 aos 60 anos, atingindo principalmente as glândulas salivares maiores, sendo a glândula parótida a mais acometida. Mas, podendo acometer também as glândulas salivares menores, no qual o palato é a região mais acometida (Gama et al., 2018).

Conforme estudo de Neville (2016), sua etiologia é decorrente de uma mistura de elementos ductais e mioepiteliais, podendo ter uma variação microscópica de um tumor para outro. Clinicamente, apresenta-se como uma massa firme, de crescimento lento e indolor.

Tipicamente, este tumor manifesta-se encapsulado e circunscrito, todavia, a cápsula pode estar incompleta ou com infiltração pelas células tumorais. Ademais, o AP é constituído por uma mistura de epitélio glandular e células mioepiteliais, dentro de um estroma parecido com o mesênquima (Porto et al., 2014).

Carvalho (2016) afirma que os métodos de diagnósticos auxiliares podem ser a sialografia, cujo é utilizado para analisar tumores de grandes dimensões, ultrassonografia para identificar se a lesão é sólida ou cística, tomografia computadorizada para tamanho preciso da lesão e ressonância nuclear magnética, para comprovar benignidade ou malignização da lesão.

Se não tratado, pode crescer a proporções grotescas. Sendo assim, a excisão cirúrgica é a melhor alternativa terapêutica, por se tratar de uma lesão benigna e a fim de evitar sua recidiva; se feita de maneira adequada, há uma incidência de cura de 95\%. No entanto, apresenta risco de complicação potencial que é o risco de malignização, podendo ocorrer em cerca de $5 \%$ dos casos (Bigueline et al., 2015; Neville, 2016). 
Sob essa ótica, o objetivo do presente estudo é relatar o caso clínico de um paciente com 64 anos diagnosticado com adenoma pleomórfico em região de mucosa jugal, bem como discutir a patogênese da lesão e o método de tratamento proposto.

\section{Metodologia}

O presente estudo se dispõe a apresentar uma descrição do diagnóstico clínico do adenoma pleomórfico, juntamente com os exames complementares histopatológicos por meio de relato de caso clínico, com objetivos descritivos e abordagem qualitativa (Pereira et al., 2018). Assim, em concordância com o conhecimento da prática estomatológica e com base na literatura científica sobre o tema abordado, e considerando a uma sequência de condução de diagnóstico do caso apresentado, propõe-se relatar um caso clínico de adenoma pleomórfico na mucosa jugal, com diagnóstico fundamentado por meio de exame clínico, em associação com a biópsia. O estudo teve o Termo de Consentimento Livre e Esclarecido - TCLE e está em apreciação pelo Comitê de Ética e Pesquisa.

\section{Relato de caso}

Paciente do sexo masculino, 64 anos, compareceu a Clínica Escola de Odontologia queixando-se de lesão assintomática em boca. Ao ser questionada informou que surgiu há aproximadamente 08 meses, evoluindo de forma lenta. Ao exame físico intraoral observou-se a presença de lesão nodular e única em mucosa jugal, bem delimitada, de coloração branca, avermelhada e amarelada, superfície lisa, formato oval, consistência firme, indolor e medindo aproximadamente 06 x 03 x 03 centímetros (Figura 1). Observou-se também a ausência dentária parcial em maxila e mandíbula e uso de prótese removível mal adaptada em arcada superior. As hipóteses de diagnóstico foram: Lipoma, lipossarcoma e adenoma pleomórfico.

Figura 1 - Lesão adenoma plemórfico.

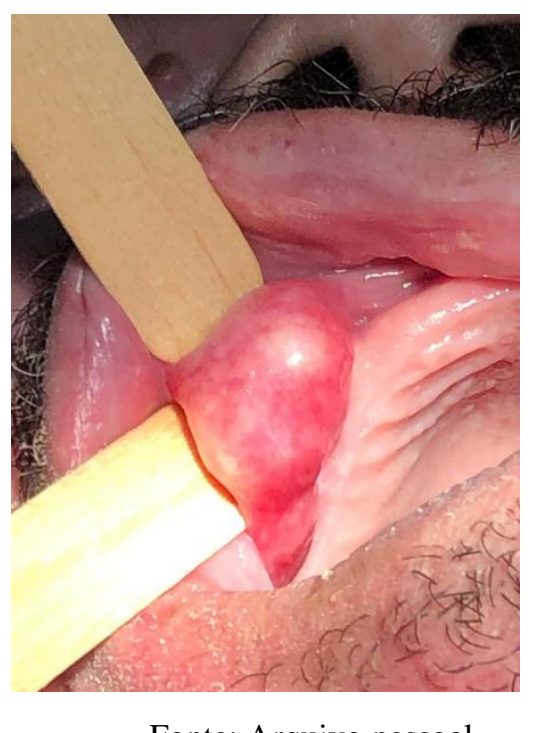

Fonte: Arquivo pessoal.

Optou-se pela realização da biópsia excisional. Inicialmente sob anestesia local na região, foi feita uma incisão em forma de cunha com uso de bisturi convencional, removendo a lesão com pequena margem de segurança (Figuras 2a-b) e posteriormente foi realizada uma sutura simples com fio de nylon 3-0 (Figura 2c). 


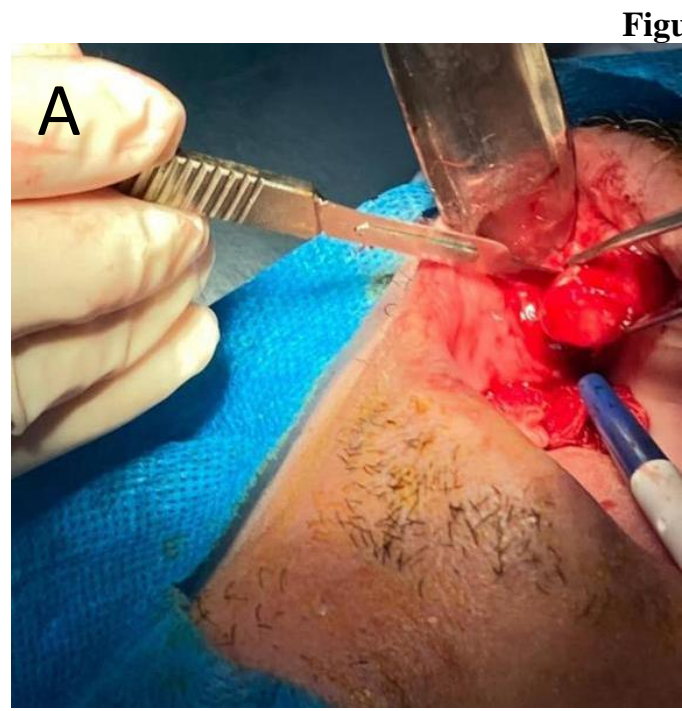

Figura 2 (A, B e C): remoção da lesão.
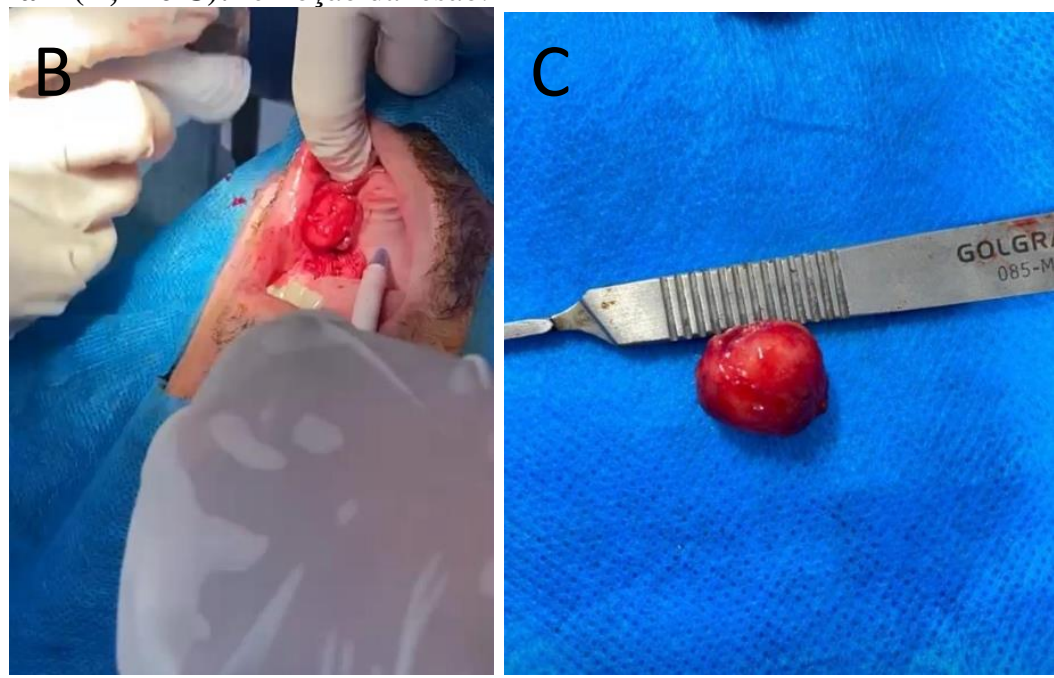

Fonte: Arquivo pessoal.

Com 08 dias de pós-operatório, realizou-se a remoção de sutura e o local apresentava aspecto clínico de cicatrização sem evidências de processo infeccioso e deiscências. O paciente foi acompanhado durante 5 meses e não apresentou sinais clínicos de recidiva da lesão (Figuras 3 a, b e c).

Figura 3 (A, B e C): 5 meses após biópsia.
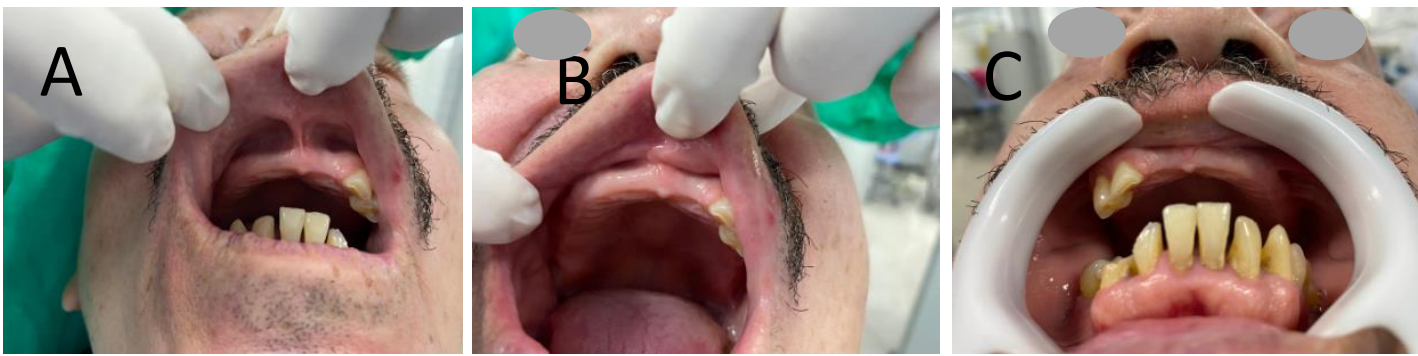

Fonte: Arquivo pessoal.

Nas Figuras 4 (A e B), pode ser observado características histopatológicas da lesão mostrando células epiteliais neoplásicas ductais formando lençóis e estruturas ductiformes e células mioepiteliais associadas a áreas hialinas e mixoides.

Figura 4 (A e B): lâminas histológicas coradas em hematoxilina e eosina evidenciando tecido epitelial e conjuntivo.
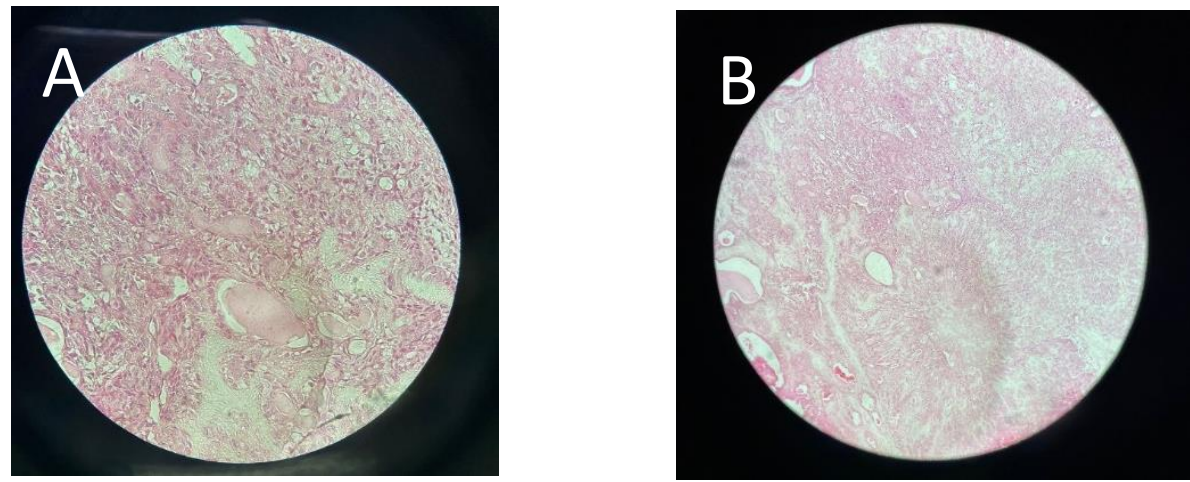

Fonte: Arquivo pessoal. 
Research, Society and Development, v. 10, n. 14, e555101422301, 2021

(CC BY 4.0) | ISSN 2525-3409 | DOI: http://dx.doi.org/10.33448/rsd-v10i14.22301

\section{Resultados e Discussão}

O adenoma pleomórfico é tido como um tumor benigno, mas pode sofrer transformações malignas, como o adenocarcinoma e o adenoma polimorfo de baixo grau (Santos et al., 2016). De acordo com Bahbah \& Chbicheb (2020), representam cerca de $50 \%$ entre os tumores das glândulas salivares menores, sento o palato o sítio anatômico mais comum (42,8 - 68,8\%), seguido do lábio superior $(10,1 \%)$ e mucosa jugal $(2,5 \%)$. Somado a isso, há outros locais raros que podem ser acometidos, como a face $(2,5 \%)$, região retromolar $(0,7 \%)$, assoalho da boca e mucosa alveolar.

Seu diagnóstico diferencial abrange outros tipos de tumores benignos, que incluem a mucocele, fibroma, lipoma, e tambéme outras neoplasias malignas como carcinoma mucoepidermóide, adenomacarcinoma, carcinoma adenomatóide cístico e carcinoma de células acinosas (Cardoso et al., 2014). No caso em questão, o exame clínico da lesão remeteu a uma lesão benigna, tendo em vista que se apresentava como uma lesão assintomática de crescimento lento, localizada em palato mole, que surgiu há aproximadamente quinze anos. Esses aspectos vão de encontro com a literatura, que confirma as características assintomáticas, tendo em vista que trata-se de neoplasia com crescimento lento (Santos et al., 2016).

Autores como Porto et al. (2014) demosntram em seu estudo que cerca de 15\% das patologias diagnosticadas predominam no sexo feminino na terceira e sexta década de vida, com uma relação de 1,5:1, o que se dá, principalmente, devido à maior busca pela estética. A localização de predileção pode acometer qualquer área da mucosa oral, sendo mais frequente em regiões como palato duro posterior e o palato mole anterior, contudo, pode ocorrer na mucosa jugal, mucosa gengival e lábios.

$\mathrm{O}$ adenoma pleomórfico pode apresentar-se bem delimitado por cápsula de tecido conjuntivo fibroso, com parênquima constituído por células epiteliais ductais e células mioepiteliais. Ainda assim, podem ser encontradas em áreas ductiformes, sólidas, mixoides, hialinas, plasmocitoides, osteoides e condroides, além de metaplasia escamosa. Comumente, manifesta-se pela presença de uma massa nodular única, firme, móvel e assintomática, contudo, seus sintomas sofrem variação conforme localização (Oliveira et al., 2016).

Autores como Bahbah \& Chbicheb (2020), descrevem a lesão com três subtipos histológicos: mixoide (estroma de 80\%), celular (células mioepiteliais predominantes) e misto (clássico). As células são constituídas em padrões de células parecidas com cordões e ductos, juntamente com áreas de metaplasia epidermóide. A matriz intracelular mostra áreas fibrosas, hialinas, mixóides, cartilaginosas e ósseas. Na região das glândulas salivares menores, a neoplasia normalmente é sólida ou celular, quando comparadas das glândulas maiores; as células mioepiteliais por sua vez, possuem citoplasma eosinófilo claro que dá fenótipo epitelioide ou plasmocitoide.

Para realização de um adequado diagnóstico é de extrema importância o exame clínico do paciente, o qual deve ser constituído pela anamnese bem detalhada, pelo exame físico e exames complementares. No decorrer do procedimento cirúrgico relatado no caso, a biopsia excisional mostrou-se um fator constituinte como exame complementar, visto que possibilitou o encaminhamento da lesão para a análise anatomopatológica, com o objetivo de obter um correto diagnóstico e um resultado de alta confiabilidade (Dutra et al., 2019; Canali et al., 2020).

Salientando a importância do tratamento de eleição, baseia-se na excisão cirúrgica com margem de segurança, uma vez que a recidiva acontece devido à permanência de resíduos da cápsula ou da própria neoplasia (de Sousa et al., 2013). Em nosso caso, o AP foi excisado ileso com sua cápsula, sendo de suma importância, pois, quando a excisão é incompleta, com ruptura da cápsula ou derramamento do tumor pode semear células tumorais nos tecidos circunvizinhos, podendo gerar sua recidiva.

\section{Conclusão}

O adenoma pleomórfico representa uma neoplasia benigna, na maioria das vezes assintomático, com isso, evidencia-se a importância do diagnóstico precoce para que ocorra tratamento, bom prognóstico e evitar sua recidiva. A biópsia excisional é 
imprescindível para diferenciar a lesão de outras patologias, constatando seu diagnóstico. A atenção profissional possibilita a resolução do problema através do tratamento adequado, assim como a orientação ao paciente.

Com uma boa proservação e o acompanhamento clínico pelo menos por cinco anos através do cirurgião-dentista capacitado, o paciente retoma sua vida e seu bem-estar normalmente, sem maiores complicações.

Para os próximos trabalhos, sugere-se a necessidade da realização de novos estudos que visem a melhor compreensão da etiologia e prevalência do adenoma pleomórfico em pacientes, embora seja um tumor benigno de glândula salivar as características clínicas dessa lesão podem ser semelhantes em tumores malignos. Sendo assim diagnósticos precoces resultam na maioria dos casos em tratamentos mais conservadores e sucesso no tratamento.

\section{Referências}

Bahbah, S., \& Chbicheb, S. (2020). Adenoma pleomórfico da bochecha: relato de caso com revisão. Int. J. Odontostomat., 14 (4).

Biguelini, G. S., Silva, S. O., Linden, M. S. S., Trentin, M. S., Miyagaki, D. C., \& Carll, J. P. (2015). Adenoma pleomórfico: características clínicas e protocolo diagnóstico. Salusvita, 34 (2).

Cardoso, C. S., Sousa, L. H. P. N., Costa, L. J., Soares, M S. M., Rosa, M. R. D., \& Bonan, P. R. F. (2014). Adenoma pleomórfico em mucosa jugal: relato de caso. Rev. Odontol. Bras. Central., 23 (65).

Canali, L. G. M., Pereira, E. S. B. M., Herculiani, P. P., Herculiani, A. P., \& Euletério, R. G. (2020). Comprometimento de papila parotídea em lesão de hiperplasia fibrosa inflamatória: relato de caso. REAOdonto, 1: e4179.

Debnath, S. C., Saika, A. K., \& Debnath, A. (2010). Pleomorphic adenoma of the palate. J Maxillofac Oral Surg., $420-423$.

Carvalho, C. Y. B., Pinheiro, F. J., Costa, F. W. G., Germano, A. R., \& Albuquerque, A. F. M. (2016). Extenso adenoma pleomorfico em região de palato: relato de caso. Jornada Odontológica dos Acadêmicos da Católica - JOAC, 2 (2).

Santos, H. K. A., Damasceno, R. V. M., Cardoso, J. A., Cancio, A. V., \& de Farias, J. G. (2016). Relatos de tratamentos distintos para o adenoma pleomórfico. Rev. Cir. Traumatol. Buco-Maxilo-Fac., 16 (3).

Dutra, K. L., Longo, L., Grando, L. J., \& Rivero, E. R. C. (2019). Incidence of reactive hyperplastic lesions in the oral cavity: a 10 year retrospective study in Santa Catarina, Brazil. Brazilian journal of otorhinolaryngology, 85, 399-407.

Farynowska, J., Błochowiak, K., Trzybulska, D., \& Wyganowska-Świątkowska, M. (2018). Retrospective analysis of reactive hyperplastic lesions in the oral cavity, European Journal of Clinical and Experimental Medicine, 16 (2), 92-96.

Gama, E. P., Vilela, D. D. C., Simões, C. C., de Farias, J. G., \& Cardoso, J. A. (2018). Adenoma pleomórfico em mucosa jugal: relato de caso clínico. Revista de Cirurgia e Traumatologia Buco-Maxilo-Facial, 18 (3), 41-44.

Knight, J., \& Ratnasingham, K. (2015). Metastasising pleomorphic adenoma: systematic review. International Journal of Surgery, 19, $137-145$.

Martins, J. C., Essvein, G., Vargas-Ferreira, F., da Silva, A. D., \& Neves, M. (2017). Prevalence of oral lesions diagnosed at the ULBRA Canoas of Dental Diagnosis Service. Stomatos, 23 (44), 24-32.

Neville, B. W., Damm, D. D., Allen, C. M., Chi, A. C. (2016). Patologia Oral e Maxilofacial. 4nd ed, Rio de Janeiro: Editora Elsevier, 928p.

Oliveira, L. J., Castro, H. H. O., Leão, P. L. R., Leal, R. M., Horta, M. C. R., \& Souza, P. E. A. (2016). Tratamento de aden oma pleomórfico em palato: relato de 2 casos e revisão de literatura. Rev. Port. Estomatol. Med. Dent. Cir. Maxilofac., 57 (1), 55-61.

Orsini, S., \& Marques, J. S. (2017). Adenoma pleomórfico. [Dissertação para obtenção de grau de Mestre em Medicina Dentária]. Instituto Superior de Ciências da Saúde Egas Moniz.

Pereira, A. S. et al. (2018). Metodologia da pesquisa científica. UFSM.

Pinto, L. G., de Figueiredo, N. F. D., Romão, T. C. M., Ferreira, L. A. B., dos Santos, M. Q., Pereira, J. A. T., Inaoka, S. D., \& Costa, D. F. N. (2020). Exérese cirúrgica de adenoma plemórfico em palato: relato de caso. Arch Health Invest, 9 (5), 449-452.

Porto, D. E., Cavalcante, J. R., Cavalcante Júnior, J. R., Costa, M. C. F., \& Pereira, S. M. Adenoma Pleomórfico de Parótida - Relato de caso. Revista de Cirurgia e Traumatologia Buco-Maxilo-Facial, 14 (2).

Silva, D. N., Guimarães, K. B., Ferraro-Bezerra, M., \& Heitz, C. (2007). Enucleação de Adenoma Pleomórfico: considerações terapêuticas e relato de caso. Revista de Cirurgia e Traumatologia Buco-Maxilo-Facial, 7 (4), 25-30.

Trindade, M. G. F., de Oliveira, M. C., do Prado, J. P., \& Santana, L. L. P. (2018). Lesões associadas à má adaptação e má higienização da prótese total. Id On Line Revista de Psicologia, 12 (42), 956-968.

Vaz, D. D. A., Valença, D. L., Lopes, R. B. D. M., Silva, A. V. C., \& Pereira, J. R. D. (2011). Concordância entre os diagnósticos clínicos e histopatológicos do Laboratório de Patologia Bucal da Faculdade de Odontologia de Pernambuco. RPG. Revista de Pós-Graduação, 18 (4), $236-243$. 